\title{
Laborübertragbare Kalibrierung von Sensoren für die Messung von Benzol
}

\author{
T. Sauerwald ${ }^{1 *}$, T. Baur 1 , M. Leidinger ${ }^{1}$, Laurent Spinelle², Michel Gerboles², A. Schütze ${ }^{1}$ \\ 1 Universität des Saarlandes/Lehrstuhl für Messtechnik, Saarbrücken/GER, \\ t.sauerwald@Imt.uni-saarland.de \\ ${ }^{2}$ European Commission, Joint Research Centre (JRC), Directorate for Energy, Transport and Climate, \\ Air and Climate Unit, Via Enrico Fermi, 2749, 21027 Ispra (VA), Italy
}

\begin{abstract}
:
Ein Sensorsystem aus drei Halbleitergassensoren mit temperaturzyklischem Betrieb wurde auf den Nachweis von Benzol im Bereich von 500 ppt bis 10 ppb bei $10-40 \%$ relativer Feuchte (rF) kalibriert und der Einfluss von Hintergrundgasen auf die Erkennung getestet. Die Kalibration zeigt eine sehr hohe Genauigkeit und Linearität, die die Interpolation von nicht kalibrierten Benzolkonzentrationen und Feuchten ermöglicht. Der Einfluss von Hintergrundgasen wie z.B. 500 ppb Wasserstoff und 150500 ppb Kohlenmonoxid kann mit Hilfe einer multilinearen Regression kompensiert werden. Das Sensorsystem wurde später in einem zweiten Gasmischsystem kalibriert und die Übertragbarkeit dieser Kalibrierung auf das ursprüngliche Gasmischsystem getestet. Der Laborvergleich zeigt eine gute Stabilität des Benzolsignals, allerdings bleibt bei der Übertragung ein Offset von ca. einem ppb zwischen den Messwerten aus den beiden Laboren. Die Ergebnisse zeigen, dass eine Übertragbarkeit zwischen Laboren im Hinblick auf eine Qualitätssicherung der Kalibrierung möglich scheint.
\end{abstract}

Keywords: Spurengasmessung, laborübertragbare Kalibrierung, Benzol.

\section{Einleitung}

Halbleiter-Gassensoren sind extrem sensitiv und können breitbandig reduzierende Gase und flüchtige organische Komponenten (volatile organic compounds - VOC) detektieren [1]. Ein oder mehrere Gase können mit Hilfe des temperaturzyklischen Betriebs selektiv identifiziert [2] und quantifiziert [3-4] werden. Eine Herausforderung ist, die Sensoren so zu kalibrieren, dass die Erkennung zuverlässig auch vor einem Hintergrund weiterer reduzierender Gas funktioniert. Dazu müssen sehr umfangreiche Testläufe mit einer Vielzahl von wohldefinierten Gaszusammensetzungen eingestellt werden, wobei die VOC-Konzentrationen im Bereich von ppb liegen [5-6]. Die Übertragbarkeit dieses Trainings bzw. dieser Kalibrierung des Systems auf Messungen im Feld ist nur zu leisten, wenn das Gasprofil die realen Bedingungen gut widerspiegelt [7-8], z. B. die Variation der Feuchte, der Konzentration von anorganischen reduzierenden Gasen [9-10] und der Konzentration der VOC [10-11]. Dennoch bleibt die Notwendigkeit, die Ergebnisse der Laborkalibirerung zu validieren. Neben der Validierung im Feld müssen auch vergleichende Tests zwischen Laboren etabliert werden, um die Kalibrierverfahren zu validieren. Ein erster Vergleich wurde mit Tests am Lehrstuhl für Messtechnik (LMT - Saarbrücken, Deutschland) und am Joint Research Center (JRC - Ispra, Italien) möglich. Die beiden Labore nutzen einen grundsätzlich anderen Ansatz der Prüfgasbereitung. Während am LMT eine kontinuierliche Gasmischung eingesetzt wird, bei der die Gaskonzentration über das Mischungsverhältnis eingestellt wird [13], [14], nutzt das JRC einen geschlossenen Regelkreis, bei dem die Gaskonzentration über ein Referenzverfahren gemessen wird [15].

\section{Experimentelles}

Für die Messungen wird ein Sensorsystem mit drei Halbleitergassensoren verwendet, das aus einem Doppelsensor (MiCS 4510, SGX SA, Schweiz) und einem Einzelsensor (AS-MLV, ams Sensor Solutions, Deutschland) besteht. Alle Sensoren werden mit einem Temperaturzyklus betrieben (Abbildung 1), der besonders die Gleichgewichtseinstellung der Oberfläche nach einer abrupten Absenkung der Temperatur nutzt. In diesem Bereich ist der Sensor sehr empfindlich auf Spuren von reduzierenden Gasen. Für die Quantifizierung wird die Steigung $d(\ln (G)) / d t$ des logarithmierten Leitwerts $G$ über die Zeit $t$ genutzt. Dieses Merkmal verhält sich in guter Näherung linear zur Konzentration des reduzierenden Gases [1]. 


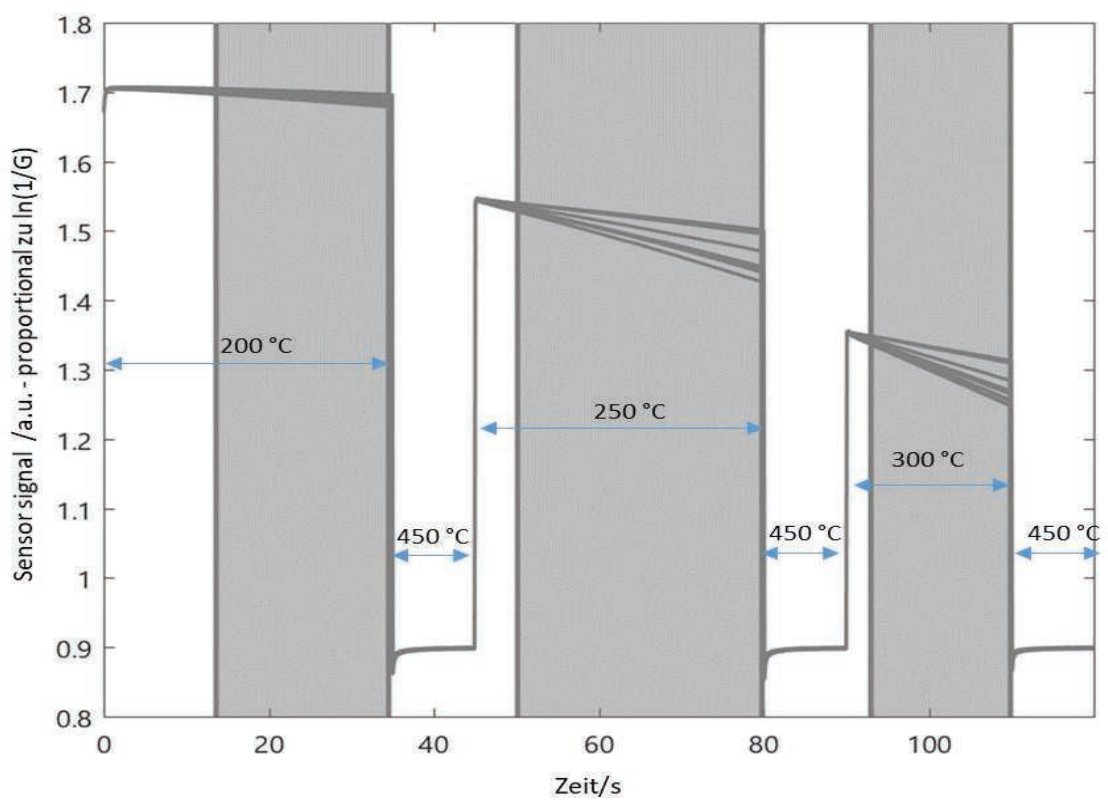

Abbildung 1: Sensorsignale im Temperaturzyklus (Temperaturbereiche: blaue Pfeile) bei verschiedenen Benzolkonzentrationen (0,5-6,5 ppb). Die grauen Bereiche werden zur Berechnung der Steigung (Merkmal) herangezogen.

Der Vergleich der Merkmale in den drei niedrigen Temperaturbereichen (Abb. 1) ermöglicht die selektive Messung von Benzol durch die Kompensation anderer Signalanteile, z.B. durch Toluol [16], indem auf Basis der Merkmale mit einer Partial Least Square Regression (PLSR) auf die Benzolkonzentration trainiert werden. Für die Einstellung der Gaskonzentration wird am LMT eine Anlage mit dynamischer Verdünnung gewählt. Bei dieser wird die Gaskonzentration über das Mischungsverhältnis von zweistufigen Verdünnungslinien eingestellt. Es findet keine kontinuierliche Rückmessung der Gaskonzentration, wohl aber eine Rückmessung der einzelnen Gasflüsse statt [5], [14], aus denen die Konzentrationen von Benzol und anderen Gasen berechnet werden. Die Einstellung der Gaskonzentration am JRC erfolgt mit Hilfe eines geschlossenen Regelkreises [15]. Für die Regelung der Benzolkonzentration wird hierbei ein PTR-MS (Proton-Transfer-ReaktionMassenspektrometer) verwendet, das die Benzolkonzentration mit hoher Zeitauflösung sampelt. Für die Annotation der Daten wird jedoch die Rückmessung mit Hilfe eines GC-PID (Gaschromatograph mit Photoionisationsdetektor) verwendet, der eine deutlich bessere Stabilität und Messgenauigkeit aufweist. Die übrigen Prozessparameter, vor allem die relative Feuchte, werden ebenfalls rückgemessen und geregelt [15].

\section{Ergebnisse}

Das Sensorsystem wurde am LMT in reiner Nullluft mit Benzolkonzentrationen von 0,5-10 ppb bei relativen Feuchten von $10 \% \mathrm{rF}, 25 \% \mathrm{rF}$ und $40 \% \mathrm{rF}$ getestet. Eine detaillierte Beschreibung der Prüfgasbereitung findet sich in [14]. Die Quantifizierung von Benzol aus den oben gezeigten Steigungssignalen (Abb. 1) erfolgte mit Hilfe einer PLSR (Partial Least Square Regression). Die PLSR ist ein Verfahren für eine multilineare Regression, das mit $0,5 \mathrm{ppb}, 3 \mathrm{ppb}$ und $10 \mathrm{ppb}$ Benzol bei $10 \% \mathrm{rF}$ und $40 \% \mathrm{rF}$ trainiert wurde. Die übrigen Messpunkte wurden in die Regression projiziert (Testdaten). Sowohl Trainings- als auch Testdaten zeigen nur sehr geringe Abweichungen von der Regressionsgerade von weniger als 0,5 ppb (Abb. 2). Dies zeigt, dass mit Hilfe dieses Verfahrens der Einfluss von Feuchte auf das Messergebnis sehr gering ist und dass sich auch unbekannte Benzolkonzentrationen sehr gut mit der Regression bestimmen (interpolieren) lassen. 


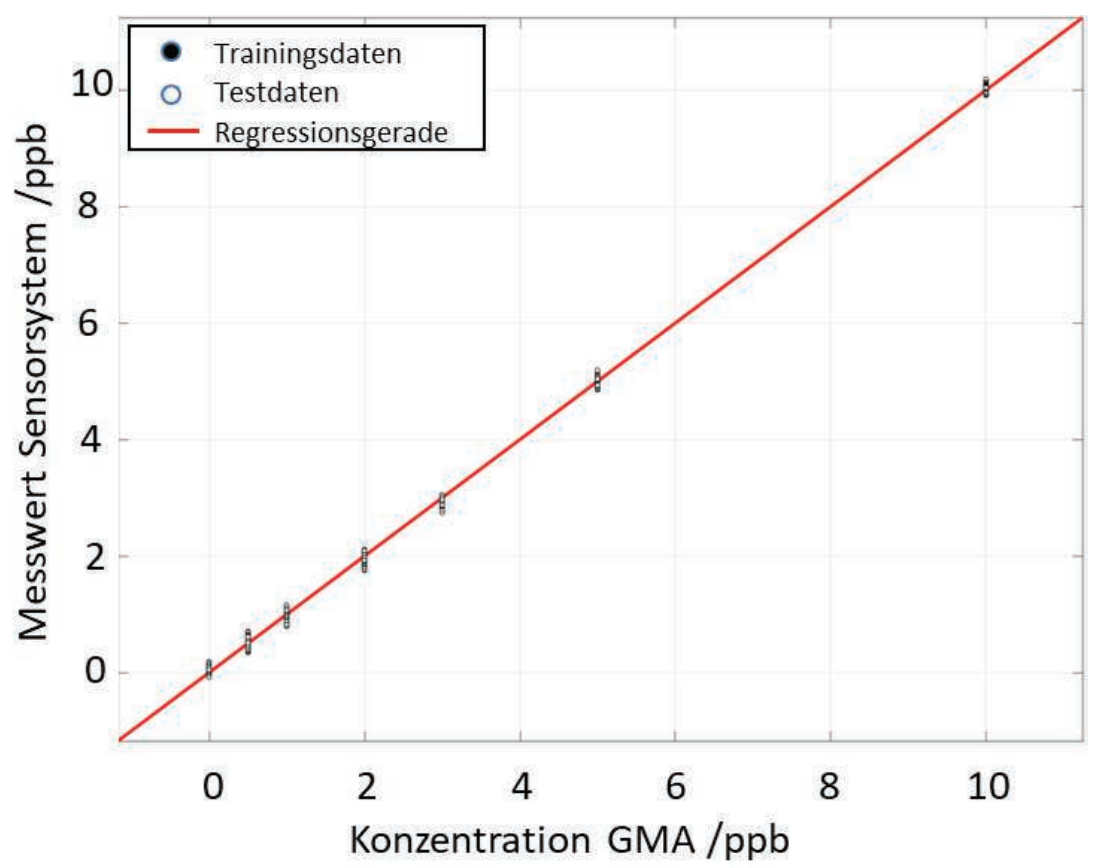

Abbildung 2: Quantifizierung von Benzol in reiner Luft bei verschiedenen Feuchten (nach [16]).

In weiteren Versuchen wurden gezielt Hintergrundgase zugefügt, um zu testen, ob der Einfluss dieser Gase mit Hilfe einer PLSR ebenfalls kompensierbar ist. Die reine Nullluft wurde dabei mit 500 ppb Wasserstoff $\left(\mathrm{H}_{2}\right), 150 \mathrm{ppb}$ Kohlenmonoxid (CO) sowie $1.820 \mathrm{ppb}$ Methan versetzt, um einen Hintergrund von sauberer Außenluft zu simulieren, in der diese Gase ubiquitär sind [9], [10]. Zusätzlich wurde ein variabler Anteil von Toluol (0 ppb, 2 ppb, 20 ppb) und eine Erhöhung der CO Konzentration auf 500 ppb getestet, um typische Störkonzentrationen zu untersuchen [12]. Die aus dem Temperaturzyklus gewonnenen Steigungsmerkmale lassen sich weiterhin gut mit einer PLSR zur Bestimmung der Benzolkonzentration auswerten. Die Streuung der Messergebnisse um die ideale Regressionsgerade ist zwar deutlich erhöht, allerdings sind die Messwerte weiterhin in guter Näherung linear zur angebotenen Benzolkonzentration (Abb. 3). 


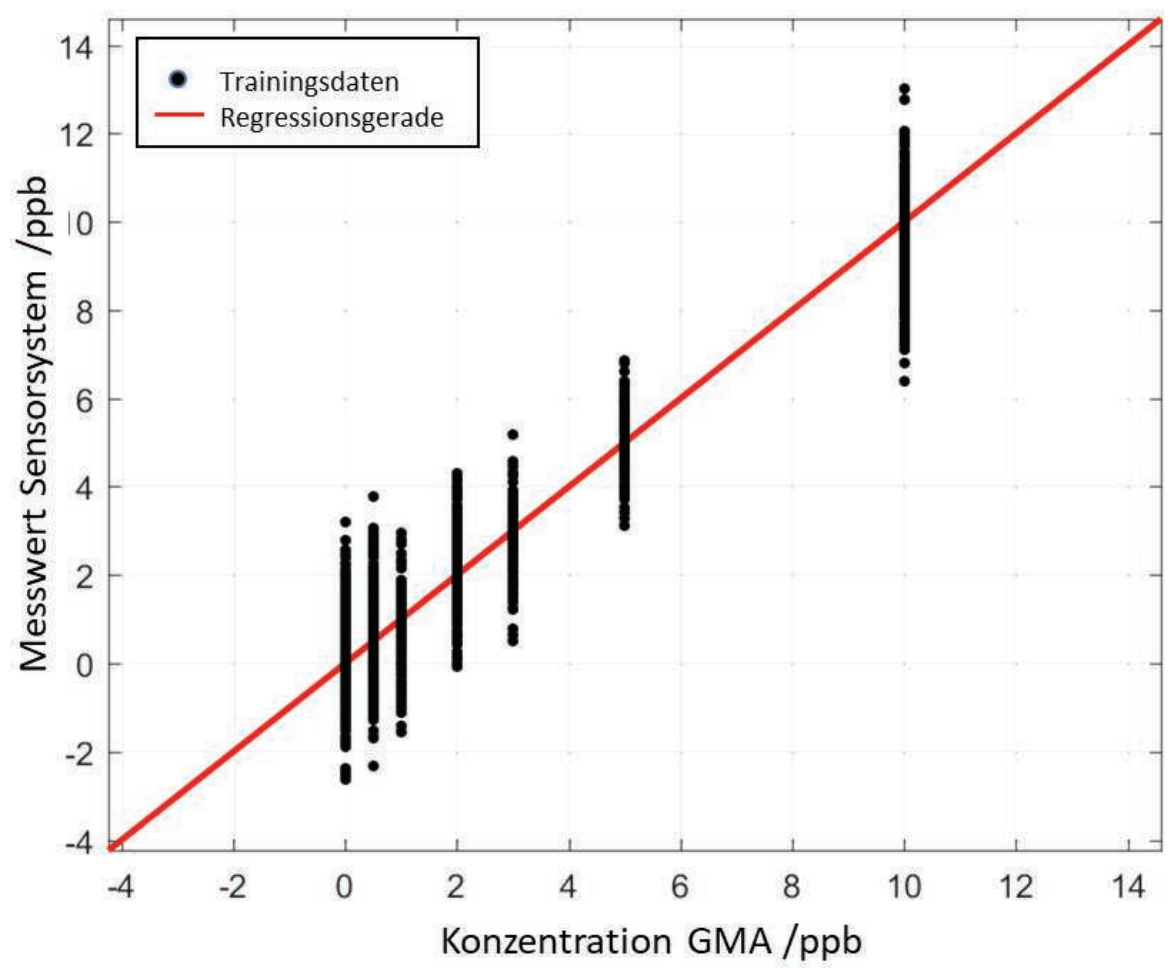

Abbildung 3: Quantifizierung von Benzol im Konzentrationsbereich von 0 bis 10 ppb mit Variation der relativen Feuchte und zusätzlichem Hintergrund von 500 ppb H2, 150 - 500 ppb CO, 1.820 ppb Methan und 0 - 20 ppb Toluol.

Im nächsten Schritt wurde das System am JRC getestet. Während des Transfers wurde die Messwertaufnahme des MiCS 4510 Sensors beschädigt, so dass für die folgenden Auswertungen ausschließlich die Daten des Sensors AS-MLV zur Verfügung standen. Daher musste eine neue Regressionsgleichung bestimmt werden. Die entsprechenden Messungen wurden für verschiedene Benzolkonzentrationen in Nullluft bei einer relativen Feuchte von $60 \%$ durchgeführt. Auch für diese Messungen zeigt die Regression der Messwerte des Sensorsystems mit den mittels GC-PID rückgemessenen Benzolkonzentrationen einen linearen Zusammenhang mit geringer Streuung um die ideale Regressionsgerade (Abbildung 4 - schwarze Datenpunkte). Die Auflösung der Benzolkonzentration ist mit der aus den Testmessungen am LMT vergleichbar. Um die Übertragbarkeit der Regression zu testen, wurden die zuvor am LMT gemessenen Daten in die Regressionsgleichung eingesetzt, die mit den neuen JRC Messungen bestimmt wurde (Abbildung 4 rote Datenpunkte). Die am LMT in reiner Nullluft gemessenen Daten lassen sich mit dieser Regression ebenfalls gut auswerten. Da die Benzolkonzentration am LMT nicht rückgemessen werden kann, wird hier die aus dem Mischungsverhältnis errechnete Benzolkonzentration gegen die durch das System bestimmte Konzentration aufgetragen. Die Messwerte sind in guter Näherung linear zu den eingestellten Konzentrationen, allerdings ist ein deutlicher Offset zu beobachten. Dieser Offset liegt im Bereich von 1-2 ppb. 


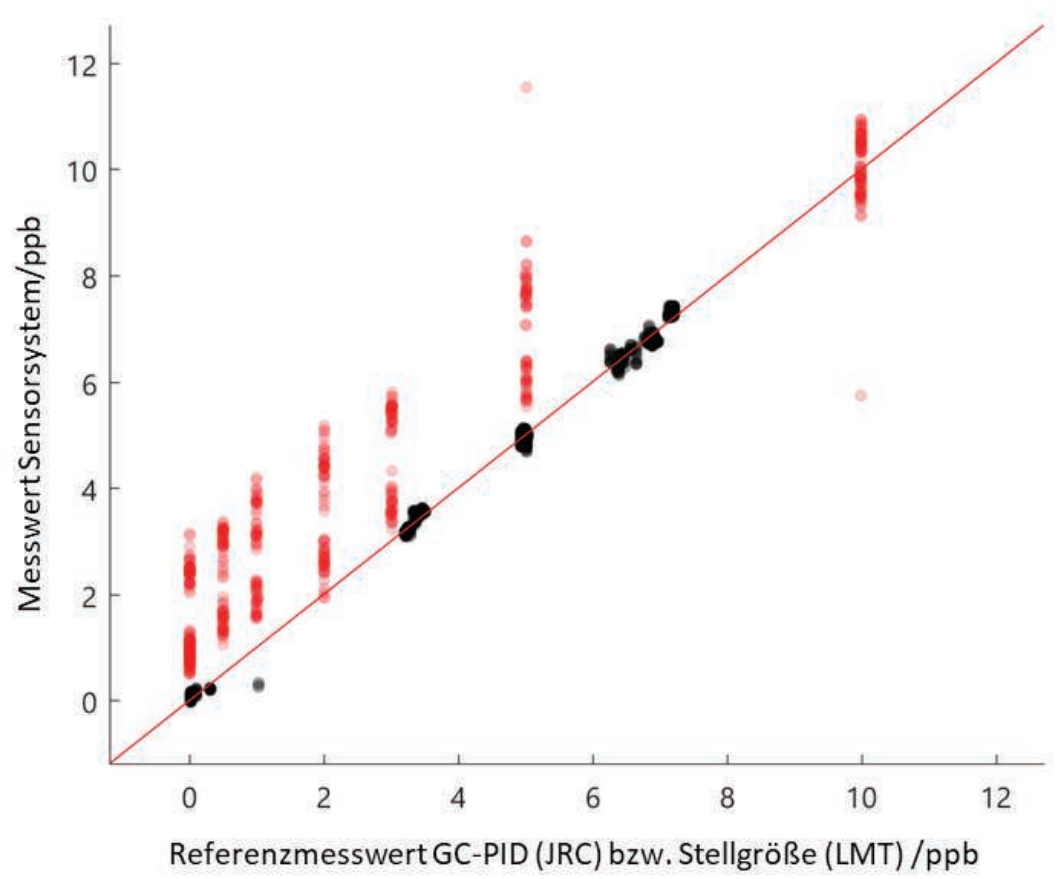

Abbildung 4: Übertragung des PLSR Modells aus der JRC Messung (schwarze Punkte) auf die Daten der LMT Messung (rote Punkte) (nach [17])

\section{Diskussion und Ausblick}

Die Daten zeigen, dass Sensorsysteme mit Halbleitergassensoren im temperaturzyklischen Betrieb sehr gut für die Messung kleiner Benzolkonzentrationen geeignet sind. Mit Hilfe der PLSR können Quereinflüsse wie Feuchte, aber auch wechselnde Toluolkonzentrationen in vergleichbarer Konzentration gut kompensiert und unbekannte Konzentrationen von Benzol gut interpoliert werden. Die Feuchtekompensation setzt die Messgenauigkeit der Systeme nicht herab, die Messunsicherheit liegt deutlich unter einem ppb. Die Kompensation anderer Gase, z. B. Toluol und CO, sowie des ubiquitären Hintergrunds aus $\mathrm{H}_{2}, \mathrm{CO}$ und Methan führen hingegen zu einer deutlich reduzierten Messgenauigkeit.

Die Übertragbarkeit der Kalibrierung zwischen den beiden Laboren ist sehr vielversprechend. Zwar gibt es einen signifikanten Offset in den Messungen, die Sensitivität auf Benzol ist in den Messungen verschiedener Labore aber vergleichbar. $\mathrm{Da}$ in den Tests keine überlappende Variation des Hintergrunds (z. B. Feuchte) angeboten wurde, konnte die Kompensation eventuell nicht optimal erfolgen. Eine andere Möglichkeit zur Erklärung des Offsets ist eine verschiedene Belastung des Hintergrunds in den beiden Anlagen. In der Gasmischanlage am LMT wird die Benzolkonzentration über das Mischungsverhältnis bestimmt und nicht kontinuierlich rückgemessen. Bisherige Rückmessungen der Anlage mittels GC/MS-Laboranalysen zeigen zwar keine Verunreinigung mit Benzol, es wurde aber eine geringe Verunreinigung mit verschiedenen flüchtigen Komponenten im Bereich weniger $\mu \mathrm{g} / \mathrm{m}^{3}$, entsprechend weniger $\mathrm{ppb}$ Toluoläquivalent, festgestellt. Die Ergebnisse zeigen, dass eine Definition von übertragbaren Testbedingungen für Gassensorsysteme zur Messung im ppb-Bereich dringend erforderlich ist, damit diese Sensorsysteme ihr hohes Potential voll ausschöpfen können.

\section{Danksagung}

Die Arbeiten wurden im Rahmen des EMRP Joint Research Project ENV56 KEY-VOCs durchgeführt. Das EMRP wird durch die Mitgliedsstaaten des EMRP innerhalb des EURAMET und durch die Europäische Union gefördert. Einige Grundlagen, besonders die Erarbeitung des Sensorsystems, wurden im Rahmen des SENSIndoor Projekts gelegt (gefördert durch das 7. Rahmenprogram unter GA Nr. 604311). 


\section{Literaturnachweis}

[1] C. Schultealbert, T. Baur, A. Schütze, S. Böttcher, and T. Sauerwald, "A novel approach towards calibrated measurement of trace gases using metal oxide semiconductor sensors," Sensors Actuators, $B$ Chem., vol. 239, 2017.

[2] M. Leidinger, T. Sauerwald, T. Conrad, W. Reimringer, G. Ventura, and A. Schütze, "Selective detection of hazardous indoor VOCs using metal oxide gas sensors," in Procedia Engineering, 2014, vol. 87

[3] T. Baur, A. Schütze, and T. Sauerwald, "Optimierung des temperaturzyklischen Betriebs von Halbleitergassensoren,” Tech. Mess., vol. 82, no. 4, pp. 187-195, 2015.

[4] M. Leidinger, J. Huotari, T. Sauerwald, J. Lappalainen, and A. Schütze, "Selective detection of naphthalene with nanostructured WO<inf $>3<$ /inf $>$ gas sensors prepared by pulsed laser deposition," $J$. Sensors Sens. Syst., vol. 5, no. 1, 2016.

[5] N. Helwig, M. Schüler, C. Bur, A. Schütze, and T. Sauerwald, "Gas mixing apparatus for automated gas sensor characterization," Meas. Sci. Technol., vol. 25, no. 5, 2014.

[6] L. Spinelle, M. Gerboles, G. Kok, S. Persijn, and T. Sauerwald, "Performance Evaluation of Low-Cost BTEX Sensors and Devices within the EURAMET Key-VOCs," MPDI Proceedings, no. Lv, pp. 30-33, 2017.

[7] A. Schütze et al., "Highly Sensitive and Selective VOC Sensor Systems Based on Semiconductor Gas Sensors: How to?," Environments, vol. 4, no. 1, p. 20, 2017.

[8] M. Leidinger, T. Sauerwald, A. Schutze, C. Alepee, and M. Rieger, "Integrated pre-concentrator gas sensor system for improved trace gas sensing performance," in Proceedings of IEEE Sensors, 2017.

[9] S. Gilge et al., "Ozone, carbon monoxide and nitrogen oxides time series at four alpine GAW mountain stations in central Europe," Atmos. Chem. Phys., vol. 10, no. 24, pp. 12295-12316, 2010.

[10] D. H. Ehhalt and F. Rohrer, "The tropospheric cycle of H2: A critical review," Tellus, Ser. B Chem. Phys. Meteorol., vol. 61, no. 3, pp. 500-535, 2009.

[11] O. Geiss, G. Giannopoulos, S. Tirendi, J. Barrero-Moreno, B. R. Larsen, and D. Kotzias, "The AIRMEX study - VOC measurements in public buildings and schools/kindergartens in eleven European cities: Statistical analysis of the data," Atmos. Environ., vol. 45, no. 22, pp. 3676-3684, 2011.

[12] E. Von Schneidemesser, P. S. Monks, and C. Plass-duelmer, "Global comparison of VOC and CO observations in urban areas," Atmos. Environ., vol. 44, no. 39, pp. 5053-5064, 2010.

[13] N. Helwig, M. Schüler, C. Bur, A. Schütze, and T. Sauerwald, "Gas mixing apparatus for automated gas sensor characterization," Meas. Sci. Technol., vol. 25, no. 5, p. 55903, 2014.

[14] M. Leidinger, C. Schultealbert, J. Neu, A. Schuetze, and T. Sauerwald, "Characterization and calibration of gas sensor systems at ppb level - a versatile test gas generation system," Meas. Sci. Technol., 2017.

[15] L. Spinelle, G. Michel, and Manuel Aleixandre, "Report of laboratory and in-situ in itu validation of microsensor sensor for monitoring ambient air pollution," 2014

[16] M. Leidinger et al., "Highly sensitive benzene detection with MOS gas sensors," Proc. AMA Conf. 2017, pp. 92-97, 2017.

[17] T. Sauerwald et al., "Highly sensitive benzene detection with metal oxide semiconductor gas sensors- an inter-laboratory comparison," J. Sensors Sens. Syst. 